\title{
Effects of Eutectic Carbide Content on Erosion Behaviors in Ductile Cast Irons
}

\author{
Cheng-Hsun Hsu' ${ }^{1}$, Sheng-Chien Chiu ${ }^{1}$, Jung-Kai $\mathrm{Lu}^{1}$ and Yih-Hsun. Shih ${ }^{2}$ \\ ${ }^{1}$ Department of Materials Engineering, Tatung University, Taipei, Taiwan 104, R.O.China \\ ${ }^{2}$ Department of Metal Mold Tao-Yen Vocational High School, Tao-Yen, Taiwan 104, R.O.China
}

\begin{abstract}
During the casting process of thin-wall parts, due to fast cooling rate or chemical composition of the ductile iron, iron carbide (cementite) may exist in the microstructure that retards the mechanical properties of the iron greatly. In this study we discuss the effects of eutectic carbide contents in relation to the cooling parameters and the erosion behavior of the erosion incident angle on the casting specimens. Different content of eutectic carbide was produced by varying the cooling rate and silicon content. Solid particle erosion tests were performed using a shot-blasting machine with angular $\mathrm{Al}_{2} \mathrm{O}_{3}$ particles. The results show that, the largest amount of eutectic carbon (plate-like) exists in the faster cooling rate and lower silicon content. The amount of pearlite and nodules counts produced at lower cooling rates also decreased accordingly. Erosion rate also was found in the same trend that higher carbide content yielded better erosion resistance and lower erosion rates, but the amount of reduction is lower than the amount of increase on the hardness level. The maximum erosion rate occurred at the incident angle equaling to $\pi / 4.5$, and the deepest erosion penetration occurred at an incident angle of $\pi / 3.6$ for four comparison specimens. The impinged surface morphologies of four specimens exhibit cutting and plowed furrows at lower incident angles, but the overlapped chippings at higher incident angles. The erosion mechanisms, at medium and high incident angle erosion, show fatigue crack and subsurface lateral crack propagation in higher eutectic carbide specimen, but platelet peeling-off and internal crack and deformation in lower eutectic carbide specimen. The test also revealed that the failure mechanism of the casting thin plates tend to be ductile-brittle fracture.
\end{abstract}

(Received October 31, 2003; Accepted January 13, 2004)

Keywords: ductile cast iron, eutectic carbide, solid particle erosion, erosion rate, mechanical property, erosion mechanism

\section{Introduction}

Ductile cast iron, which possesses excellent mechanical properties, good castability and low cost, has been widely used in industrial applications where the material often exposed to erosion wear of fluid and/or solid particles, such as in piping and hydraulic valve. The erosion problem often affects the efficiency and control accuracy in many facilities. In general, as-cast microstructure of ductile iron consists of nodular graphite surrounded by a mixed matrix of ferrite and pearlite (so called bull's eye structure). Other constituents such as carbide, martensite, bainite, or austenite may exist in the matrix depending on the cooling rate, heat treatment and alloying elements. Specially, in the thin sections of tubular products, owing to the rapid cooling rate or the improper chemical composition, microstructure of the iron often found the existence of eutectic carbide. The compound, also called cementite, is relatively hard and brittle and is generally considered to be a harmful constituent in normal microstructure of ductile iron. ${ }^{1,2)}$ Some researchers ${ }^{3-14)}$ have documented the erosion mechanisms of various metallic materials using mechanics analysis. However, the presence of such hard phase, cementite, whether aids ductile iron in erosion resistance is yet to be quantified. Because of the complexity of ductile iron's microstructure, including the nodular graphite which is virtually having no strength and incoherent with matrix, beset by the bull-eye's structure, plus various cementitic contents changed with cooling rate and other factors, make it a very interesting subject to look into its erosion controlling parameters. Therefore, the purpose of this study is to explore the effect of eutectic carbide content on erosion behaviors of the ductile irons using various cooling rates and silicon contents.

\section{Experimental Procedures}

\subsection{Experimental material}

In this experiment, 4-mm and 7-mm thick ductile iron casting plates were produced using regular foundry practices. The composition and the added amount of nodularizer and inoculant are listed in Table 1. To obtain different amount of iron carbides in material design, the metallic and green sand molds were used to pour the testing plates, and some of green sand molds were controlled for early shake-out so that the change of cooling rate could be obtained. Because silicon element is a potent graphitizer, its content may affect the existence of eutectic carbide in as-cast ductile iron. Thus, the addition of silicon amount was also controlled at $2.2 \%$ and $3.0 \%$ in the study. Table 2 shows the chemical compositions of the resulting materials analyzed with a glow discharge spectrometer (GDS).

Table 1 Chemical Compositions of Nodularizer and Inoculant.

\begin{tabular}{cccccccc}
\hline & Addition & $\mathrm{Si}$ & $\mathrm{Mg}$ & $\mathrm{Ca}$ & R.E. & $\mathrm{Al}$ & $\mathrm{Fe}$ \\
\hline Nodularizer & 1.1 & 45.27 & 4.93 & 2.31 & 1.98 & 0.36 & bal. \\
Inoculant & 0.3 & 71.53 & - & 0.96 & - & 2.43 & bal.
\end{tabular}

Table 2 Chemical Compositions of the Experimental Materials.

\begin{tabular}{ccccccccc}
\hline Type of mould & $\mathrm{C}$ & $\mathrm{Si}$ & $\mathrm{Mn}$ & $\mathrm{P}$ & $\mathrm{S}$ & $\mathrm{Mg}$ & $\mathrm{Fe}$ & $\mathrm{C} . \mathrm{E}$. \\
\hline \multirow{2}{*}{ Metal } & 3.78 & 2.2 & 0.271 & 0.068 & 0.022 & 0.025 & bal. & 4.5 \\
& 3.79 & 3.0 & 0.248 & 0.063 & 0.022 & 0.032 & bal. & 4.8 \\
Sand & 3.78 & 2.2 & 0.250 & 0.052 & 0.017 & 0.024 & bal. & 4.5 \\
(Shake-out fast) & 3.78 & 3.0 & 0.250 & 0.047 & 0.015 & 0.034 & bal. & 4.8 \\
Sand & 3.74 & 2.2 & 0.235 & 0.052 & 0.017 & 0.028 & bal. & 4.5 \\
(Shake-out slow) & 3.75 & 3.0 & 0.210 & 0.050 & 0.017 & 0.033 & bal. & 4.8 \\
\hline
\end{tabular}


Table 3 The matrix constituents and hardness of the experimental materials in different thickness.

\begin{tabular}{|c|c|c|c|c|c|c|c|c|c|}
\hline \multirow{2}{*}{$\begin{array}{l}\text { Thickness } \\
(\mathrm{mm})\end{array}$} & \multirow{2}{*}{$\begin{array}{l}\text { Type } \\
\text { of } \\
\text { mold }\end{array}$} & \multirow{2}{*}{$\begin{array}{c}\mathrm{Si} \\
(\text { mass \%) }\end{array}$} & \multicolumn{3}{|c|}{ Matrix (Vol\%) } & \multirow{2}{*}{$\begin{array}{c}\text { Nodularity } \\
(\%)\end{array}$} & \multirow{2}{*}{$\begin{array}{c}\text { Nodules } \\
\text { count } \\
\left(\mathrm{No} / \mathrm{mm}^{2}\right)\end{array}$} & \multirow{2}{*}{$\begin{array}{l}\text { Brinell } \\
\text { hardness } \\
(\mathrm{BHN})\end{array}$} & \multirow{2}{*}{$\begin{array}{c}\text { Non-notch } \\
\text { impact } \\
\text { value }\left(\mathrm{J} / \mathrm{cm}^{2}\right)\end{array}$} \\
\hline & & & Carbide & Pearlite & Ferrite & & & & \\
\hline \multirow{4}{*}{$4 \mathrm{~mm}$} & Metal & 3.0 & 68 & 10 & 22 & 100 & 250 & 635 & 7 \\
\hline & $\begin{array}{l}\text { Sand } \\
\text { (Fast) }\end{array}$ & 2.2 & 10 & 45 & 45 & 100 & 190 & 219 & 20 \\
\hline & \multirow{2}{*}{$\begin{array}{l}\text { Sand } \\
\text { (Slow) }\end{array}$} & 2.2 & 2 & 48 & 50 & 100 & 130 & 202 & 23 \\
\hline & & 3.0 & 2 & 18 & 80 & 100 & 150 & 196 & 23 \\
\hline \multirow{5}{*}{$7 \mathrm{~mm}$} & \multirow{2}{*}{ Metal } & 2.2 & 51 & 29 & 20 & 90 & 220 & 386 & 13 \\
\hline & & 3.0 & 42 & 22 & 36 & 100 & 230 & 384 & 15 \\
\hline & $\begin{array}{l}\text { Sand } \\
\text { (Fast) }\end{array}$ & 2.2 & 3 & 44 & 53 & 100 & 120 & 217 & 53 \\
\hline & \multirow{2}{*}{$\begin{array}{l}\text { Sand } \\
\text { (Slow) }\end{array}$} & 2.2 & 1 & 14 & 85 & 100 & 100 & 145 & 70 \\
\hline & & 3.0 & 1 & 7 & 92 & 100 & 135 & 144 & 65 \\
\hline
\end{tabular}

\subsection{Microstructural analysis and mechanical testing}

Optical microscopy (OM) was performed to examine the material's microstructure. The amount of eutectic carbide was measured using the software of Optimas/Optimate version 6.2. Scanning electron microscopy (SEM) was applied in order to observe the surface and subsurface of eroded specimens for the evaluation of the fracture mechanism. Brinell hardness tester with $3000 \mathrm{~kg}$ load was used to provide supplemental data for the specimens. Five readings were taken and averaged to represent the hardness of the specimens in each condition. Impact testing was performed using a Charpy impact tester. The specimen is of an unnotched type as set forth in ASTM-A327 $7^{15)}$ standard. Both of the hardness and impact testing were performed in ambient environment.

\subsection{Erosion testing}

In this experiment, dry solid particle erosion test was adopted to evaluate the behaviors of erosion wear in ductile irons. The dimensions of the testing specimens were cut and machined to two different sizes of $65 \mathrm{~mm} \times 35 \mathrm{~mm} \times 3 \mathrm{~mm}$ and $35 \mathrm{~mm} \times 35 \mathrm{~mm} \times 3 \mathrm{~mm}$. The former size was used for the high incident angle $(\pi / 2, \pi / 2.25, \pi / 2.7, \pi / 3)$ tests and the latter for the low incident angle $(\pi / 18, \pi / 9, \pi / 6, \pi / 4.5$, $\pi / 3.6$ ) tests. The surface roughness of $0.1 \mu \mathrm{m}$ (Ra) was achieved on the test specimen using \#1200 waterproof abrasive paper. Angular $\mathrm{Al}_{2} \mathrm{O}_{3}$ particles (about $177 \mu \mathrm{m}$ in size and Mohr 7 scale) were selected as the eroding carriers. The amounts of erosion particles were $5 \mathrm{~g}$ and $8000 \mathrm{~g}$, respectively. The $5 \mathrm{~g}$ erosion particles is designed to impose a single particle erosion on the surface of the specimen for understanding the erosion mechanism where the $8000 \mathrm{~g}$ erosion particles is for quantifying the erosion rate. The erosion pressure was maintained at a constant value of $3 \mathrm{~kg} /$ $\mathrm{cm}^{2}$ using an air compressor. The average particle velocity at a distance from the nozzle tip of $30 \mathrm{~mm}$ (practical erosion distance) is $83.2 \mathrm{~ms}^{-1}$. ${ }^{16)}$ The internal diameter of employed nozzle is $\phi 5 \mathrm{~mm}$ and the incident angles are from $\pi / 18$ to $\pi / 2$ at an interval of $\pi / 18$.

Erosion rate is defined as the loss of mass per total weight of shot-blasting solid particles. ${ }^{17,18)}$ The weight loss was measured using a microbalance with an accuracy of $1 \times 10^{-3}$ gram. In addition, the penetrating depth of damaged specimens after erosion testing was measured using a noncontact CNC laser measurement machine. Three tests were done and averaged to represent the experimental data in each condition. Single particle erosion was also performed to assist understanding the wear mechanisms. ${ }^{15,19-22)}$

\section{Result and Discussion}

\subsection{Microstructure and erosive specimens selection}

The molding process, casting plate thickness, microstructural constituent, hardness and impact value of all experimental materials are listed in Table 3. The results showed that eutectic carbide content increased with the increase of cooling rate, i.e., from green sand mold to metallic mold and/ or from thick $(7 \mathrm{~mm})$ to thin $(4 \mathrm{~mm})$ casting plates. At the same cooling rate, it seems that the high silicon content (3.0\%) did not significantly change the percentage of eutectic carbide; however, it decreased the pearlitic content and increased ferritic content and nodules count. Silicon element, thus, plays an important role in promoting graphitization where, silicon's graphitizing power overcomes the pearlite stabilizing power of this relatively high phosphorus content. Also, hardness and impact toughness of the irons is influenced by eutectic carbide content, that is to say, the higher content of eutectic carbide, the higher hardness value and the lower impact toughness. Based on the eutectic carbide content, the resulting irons were quite easily divided into two portions of high carbide content (42 77\%) and low carbide content $(1 \sim 10 \%)$. From all of the experimental materials, four representing specimens $(3,10,42,68 \%$ carbide content) were selected for further erosion tests. Microstructures of these specimens are depicted in Fig. 1. They all showed nodularity over $100 \%$ and the plate-like eutectic carbide can be seen clearly in Figs. 1(a), (b), (c), except (d) only a few carbides was found in the matrix. The reason that metallic mold produces the cast iron with higher eutectic carbide and nodule is mainly because of its higher 

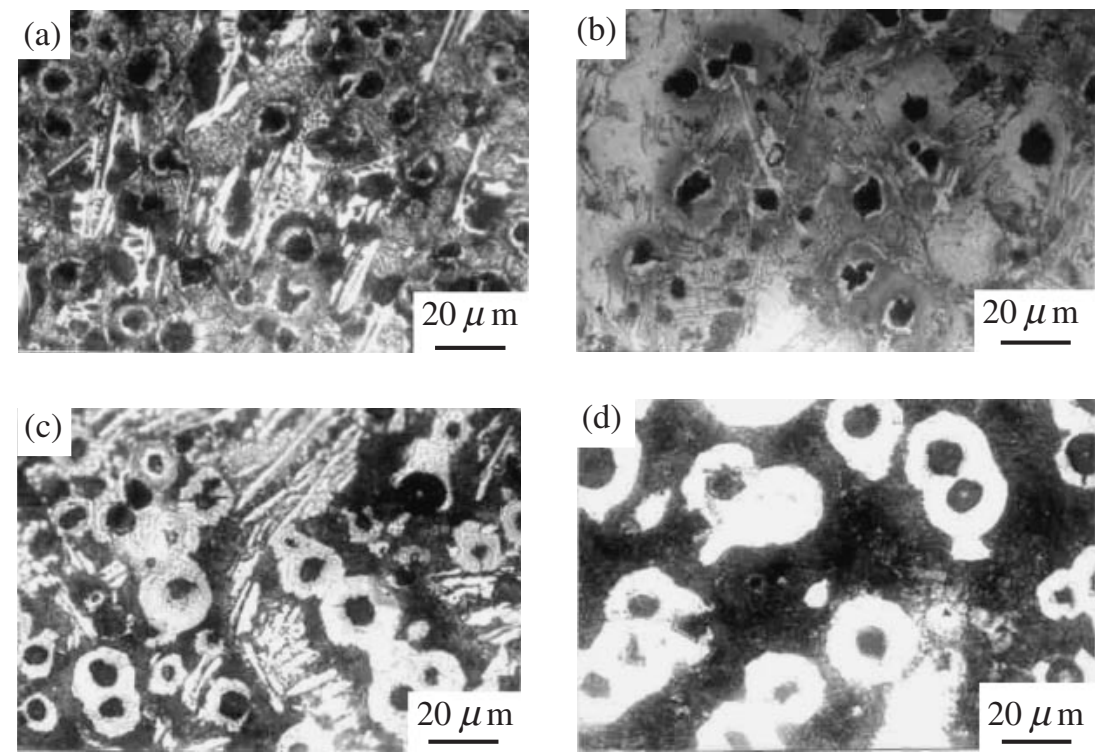

Fig. 1 Microstructures of the experimental materials for the different eutectic carbides contents specimens: (a) $68 \%$ (b) $42 \%$ (c) $10 \%$ (d) $3 \%$.
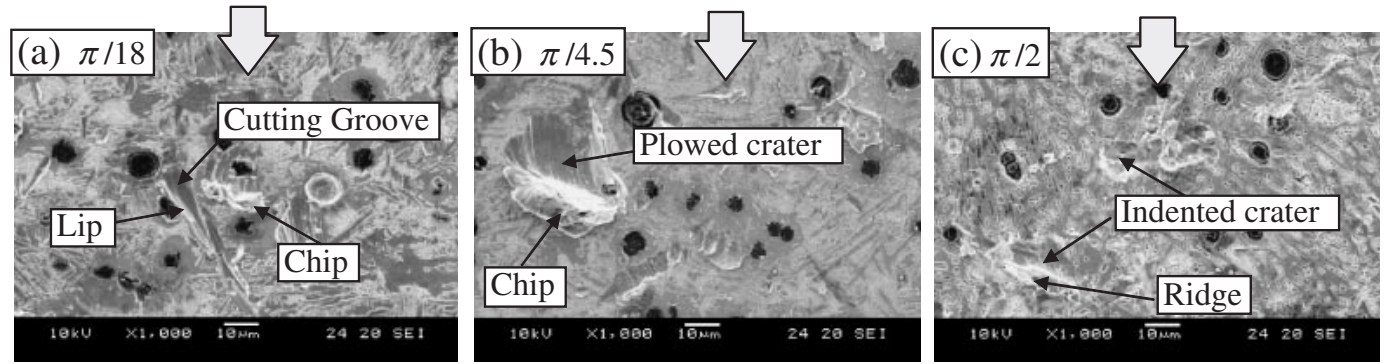

Fig. 2 SEM observation of on (a) $\pi / 18$, (b) $\pi / 4.5$, and (c) $\pi / 2$ impacted wear surface of 68 vol.pct specimens by single particle erosion,

where the arrows indicate incident direction projected on surface.

cooling rate as evidenced by the eutectic carbide content $(68 \% / 42 \%$ v.s. $10 \% / 3 \%)$ and nodule counts $(250 / 230$ v.s. 190/120). The thinner castings also resulted in a higher carbide content is due to the same reason. The average hardness $(\mathrm{BHN})$ of the test specimens are 635, 384, 219, and 217 , respectively, those may mostly reflect the amount of eutectic carbide from high to low in sequence.

On the other side, it is generally believed that the existence of ferrite in the matrix vs. cooling rates behaves in the opposite way of the carbides. However, the amount of pearlite, which normally made up the balance of the constituents besides ferrite in the regular ferrite-pearlitic matrix of ductile iron, did not seem to behave this way with the presence of carbides. While, the amount of pearlite in the mixed carbide-pearlite-ferritic matrix increased as the eutectic carbides decreased, so did ferrite. ${ }^{1,23,24)}$ This means that carbides really act as the carbon-sink that when the amount of eutectic carbides increased the presence of pearlite and ferrite decreased simultaneously. As the cooling rate decreased, besides the decrease of carbide formation and increase of ferrite content, graphite nodular size also increased and the "bull's eye" structure becomes more prominent as well. This phenomenon can also be clearly seen in Fig. 1. This is the tendency of graphite nucleation and growth during solidification process of the iron melt that graphite nodules once formed at a higher temperature has more time to grow to a large size when the cooling rate is slower.

\subsection{Erosion wear \\ 3.2.1 Single particle erosion mechanism}

Figures 2 and 3 illustrate SEM micrographs of the different carbide content specimens $(68 \%$ and $3 \%)$ at $\pi / 18, \pi / 4.5$, and $\pi / 2$ incident angles, respectively, by the single solid particle test. Comparing with the hardest $(68 \%)$ specimen, the softest (3\%) one shows wide, large grooves on the softer matrix at low incident angle (Fig. 2(a) v.s. Fig. 3(a)). In both figures, the lip can be seen. When the medium incident angle was used, the softest specimen shows a mixture of smear craters with cutting (Fig. 3(b)) and a bigger lip in front of the smear crater than the hardest specimen (Fig. 2(b)). High incident angle $(\pi / 2)$ erosion caused indentation-like craters, with a ridge being extruded at the back of the craters (Fig. 2(c) and Fig. 3(c)). Therefore, the erosion mechanism showed the tendency from cutting to mixture of cutting and smear crater, to indentation-like craters with an increasing normal force.

\subsubsection{Erosion rate and penetration depth}

To quantify the erosion rate and penetration depth, dry shot-blasting of $8000 \mathrm{~g}$ alumina particles onto testing material plates were performed at different impact angles and different eutectic carbide contents ranging from $68 \%$ to 

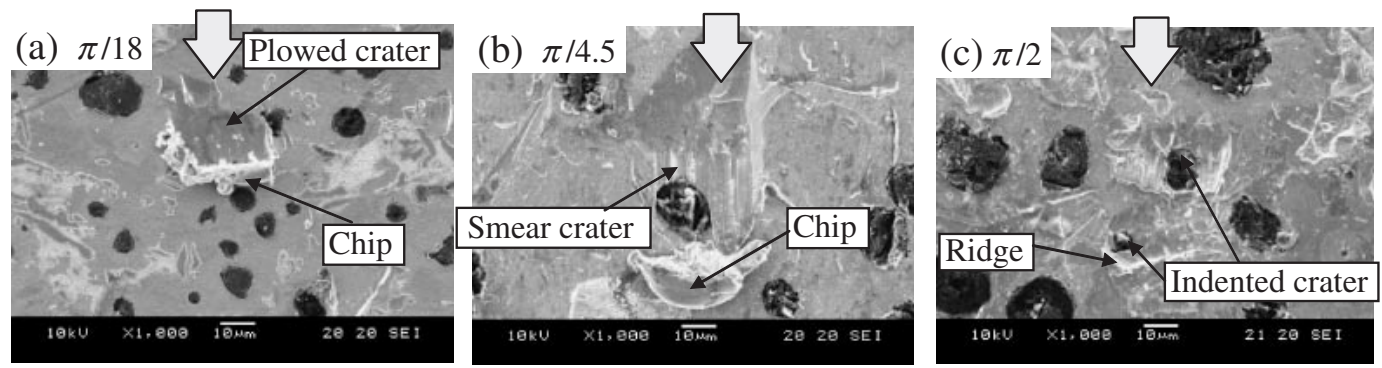

Fig. 3 SEM observation on (a) $\pi / 18$, (b) $\pi / 4.5$, and (c) $\pi / 2$ impacted wear surface of 3 vol.pct specimens by single particle erosion, where the arrows indicate incident direction projected on surface.

Table 4 Erosion rate and erosion penetrant depth for the resulting materials.

\begin{tabular}{|c|c|c|c|c|c|c|c|c|}
\hline \multirow{2}{*}{$\begin{array}{l}\text { Iron carbide content } \\
\text { Erosion angle }\end{array}$} & \multicolumn{2}{|c|}{68 vol $\%$} & \multicolumn{2}{|c|}{$42 \mathrm{vol} \%$} & \multicolumn{2}{|c|}{10 vol $\%$} & \multicolumn{2}{|c|}{$3 \mathrm{vol} \%$} \\
\hline & Rate, $\mathrm{g} / \mathrm{kg}$ & Depth, mm & Rate, $\mathrm{g} / \mathrm{kg}$ & Depth, mm & Rate, $\mathrm{g} / \mathrm{kg}$ & Depth, mm & Rate, $\mathrm{g} / \mathrm{kg}$ & Depth, mm \\
\hline$\pi / 18$ & 0.0673 & 0.29 & 0.0743 & 0.31 & 0.1038 & 0.36 & 0.1067 & 0.35 \\
\hline$\pi / 6$ & 0.1125 & 0.55 & 0.1191 & 0.57 & 0.1396 & 0.69 & 0.149 & 0.72 \\
\hline$\pi / 4.5$ & 0.1138 & 0.71 & 0.1209 & 0.78 & 0.1514 & 0.86 & 0.1518 & 0.89 \\
\hline$\pi / 3.6$ & 0.1034 & 0.77 & 0.1129 & 0.85 & 0.1361 & 0.97 & 0.1333 & 1.01 \\
\hline$\pi / 3$ & 0.0881 & 0.76 & 0.0989 & 0.79 & 0.1105 & 0.94 & 0.1128 & 0.98 \\
\hline$\pi / 2.7$ & 0.072 & 0.67 & 0.079 & 0.67 & 0.0821 & 0.65 & 0.0887 & 0.73 \\
\hline$\pi / 2.25$ & 0.0689 & 0.52 & 0.066 & 0.49 & 0.0687 & 0.53 & 0.0682 & 0.48 \\
\hline$\pi / 2$ & 0.1039 & 0.57 & 0.092 & 0.59 & 0.0997 & 0.58 & 0.0883 & 0.55 \\
\hline
\end{tabular}

(a)

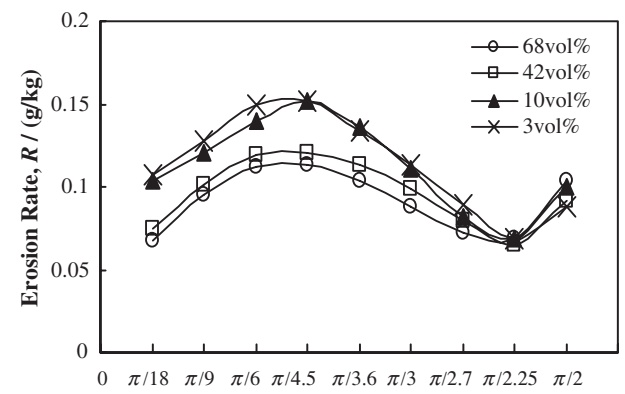

Erosion Incident Angle, $A$ / rad

(b)

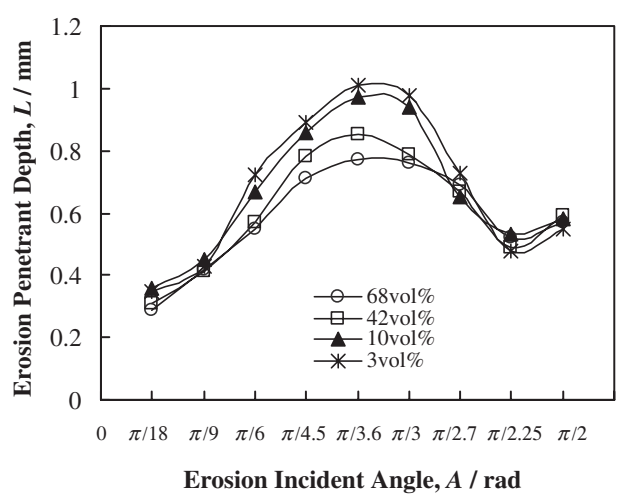

Fig. 4 The erosion loss of different eutectic carbides contents specimens test on different incident angle (a) erosion rate, (b) erosion penetrant depth

$3 \%$. The average data of erosion rate and penetration depth are depicted in Table 4 and Fig. 4. The maximum erosion rate of four test plates occurred at an incident angle of $\pi / 4.5$, as shown in Fig. 4(a) and the deepest penetration depth occurred during an incident angle of $\pi / 3.6$, as shown in Fig. 4(b). It

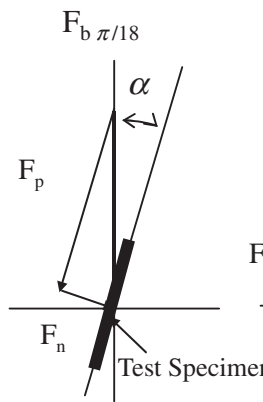

(a)

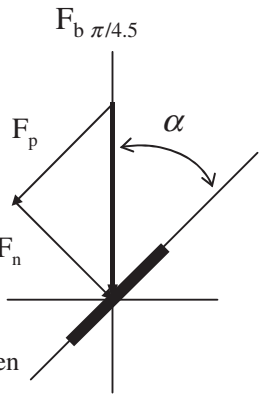

(b)

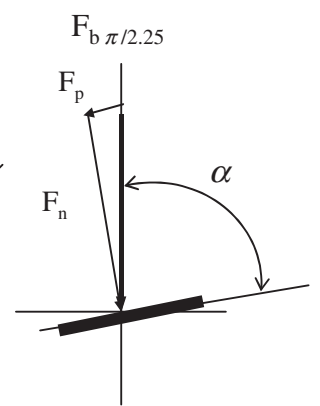

(c)
Fp : impact force parallel to test specimen (shear force)

Fn : impact force normal to test specimen

Fig. 5 The relationship between impact force components and impact angle (a) $\alpha=\pi / 18$, (b) $\alpha=\pi / 4.5$, (c) $\alpha=\pi / 2.25$.

can be seen that specimens with higher amounts of carbides content rendered lower erosion rate at all incident angles. All specimens also exhibited increasing erosion rate as the incident angle increased from $\pi / 18$ (start-up angle) to $\pi / 4.5$ then started to decrease up to $\pi / 2.25$ before the rate picked up again. This behavior was explained in the followings and shown in Fig. 5.

Shot-blasting impact force, $\overrightarrow{F b}$, can be decomposed into two force components $\overrightarrow{F n}$ and $\overrightarrow{F p}$, where $\overrightarrow{F n}$ is the force component normal to the specimen surface, and $\overrightarrow{F p}$ is the force component parallel to the specimen surface. The function of $\overrightarrow{F n}$ is to create the erosion wear of the workpiece while $\overrightarrow{F p}$ washes away the debris at the blasted surface. The $\mathrm{Al}_{2} \mathrm{O}_{3}$ particle allows gradual erosion of the specimen 
surface, which forms a new surface for the subsequent particle to work on.

When incident angle $\alpha$ increased from $\pi / 18$ to $\pi / 4.5, \overrightarrow{F n}$ also increased which causes the erosion rate to increase accordingly. Above $\pi / 4.5, \overrightarrow{F p}$ is not large enough to wash away the debris and possibly causing the particles to pile up. The pile up of debris virtually acted as a shield for preventing the workpiece for the subsequent particles from hitting the workpiece. The local shielding helped reducing the erosion rate starting from the angle at about $\pi / 4.5$ gradually reducing it to an angle of about $\pi / 2.25$. Beyond that, the normal force $\overrightarrow{F n}$ was so great as to break through all barrier debris or residual particles, which might be covering the top of the workpiece surface. New surfaces continued to be eroded thus the erosion rate started to increase again up to $\pi / 2$. Over and beyond $\pi / 2$ of incident angle, erosion occurred on the opposite direction of the workpiece, repeating the above erosion process. Apparently, high carbide content has contributed to this effect as opposed to the observation at low carbide content above.

\subsubsection{Effects of hardness and toughness}

Figures 6 and 7 illustrate the effects of hardness and impact toughness on erosion behaviors (max. of erosion rate and penetrative depth) of the ductile irons. The results showed that the tendency of the maximum erosion rate reduced with the increase of hardness value and the decrease of impact toughness. The change of the maximum penetration depth in relation to the incident angle is the same as that of the maximum erosion rate. It implies that the higher hardness value and/or the lower impact toughness, the better erosion wear resistance for ductile irons.

From the testing results, we can draw the conclusion that the hardness variation is significant and the response of the erosion rate to the impact angle showed the same tendency among the four tested specimens with the maximum erosion rate almost all occurred between the impact angles of $\pi / 6$ to $\pi / 4.5$. However, the erosion rate $(E)$ varies significantly among the four test specimens. The difference in erosion rate is especially profound in the low to medium impact angles ( $\pi / 18$ to $\pi / 3$ ), which according to the condition of the erosion test set up in our experiment indicating that the effect of the impact angles to the erosion rate is significantly higher than the effect of the material variation, i.e. the matrix constituents and hardness $(H)$. This finding is similar to Hansen et al's ${ }^{25-27)}$ where $E$ and $H$ are not proportional to each other.

\subsection{Eroded surface morphology \\ 3.3.1 Surface appearance}

Figure 8 illustrates eroded surface SEM micrographs of the two specimens $(68 \%$ and $3 \%)$ at low incident angles $(\pi / 18)$ using $8000 \mathrm{~g}$ particles erosion. It can be seen that the results were similar to the single particle erosion test, and surface appearance demonstrated a lot of micro-plowing and microcutting with predominately groove at lower impinging angles, and indented crater and overlapped chippings at higher impinging angles. Figure 8(a), the highest carbide content, shows that the eroded surface of the material was

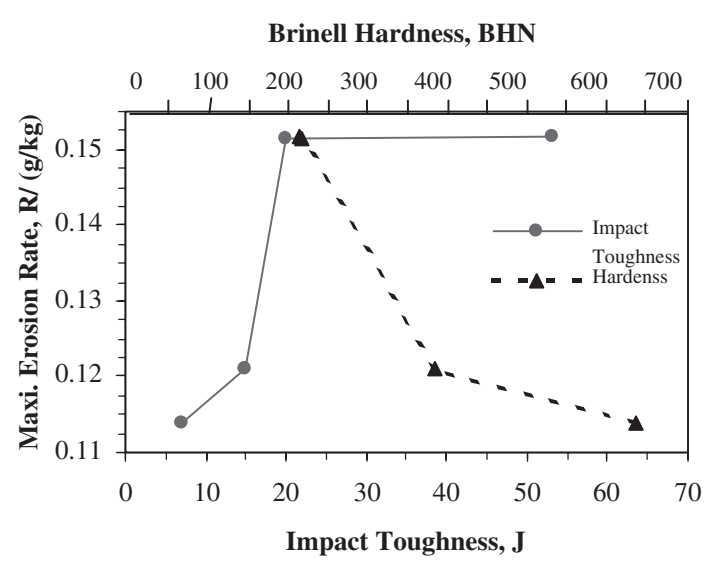

Fig. 6 The relationship of maximum erosion rate on (a) Impact value, (b) Hardness, for four tested samples.

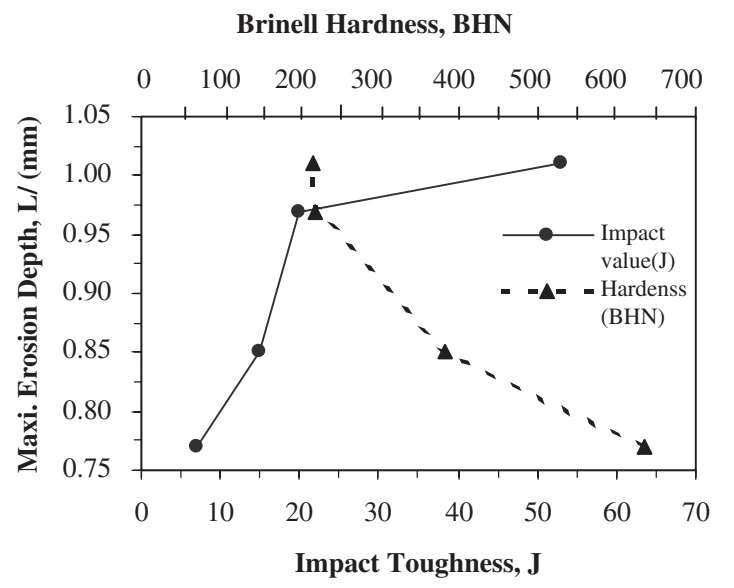

Fig. 7 The relationship of maximum erosion depth on (a) Impact value, (b) Hardness, for four tested samples.

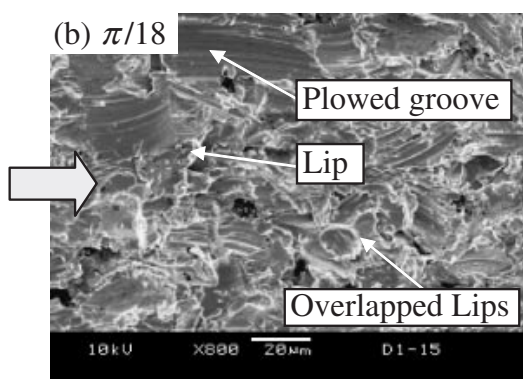

Fig. 8 SEM observation on low incident angle impacted wear surface of (a) $68 \%$ (b) $3 \%$ specimens by $8000 \mathrm{~g}$ repetitious erosion, where the arrows indicate incident direction projected on surface. 

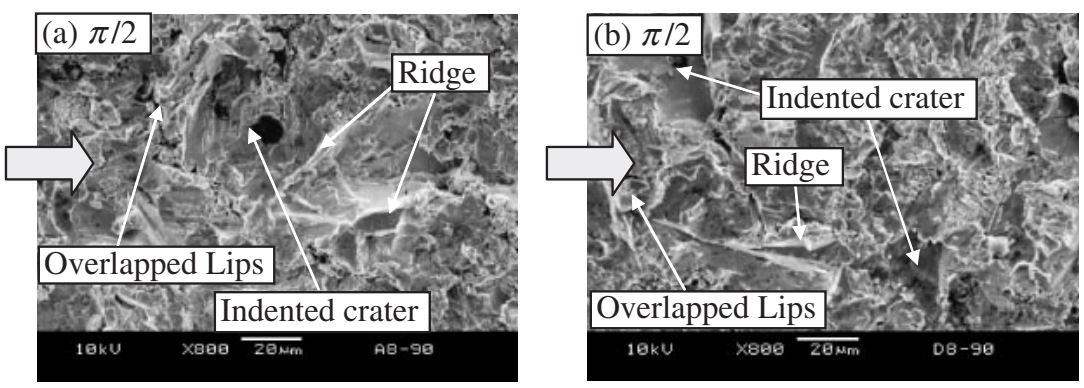

Fig. 9 SEM observation on high incident angle impacted wear surface of (a) 68\% (b) $3 \%$ specimens by $8000 \mathrm{~g}$ repetitious erosion.

plowed by the incident particles then detached. In contrast, the specimen of the lowest hardness shows that the eroded surface had signs of plastic deformation prior to detaching, as seen in Fig. 8(b). Figure 9 illustrates the eroded surfaces of the two specimens $(68 \%$ and $3 \%)$ at a high incident angle of $\pi / 2$, where indentation-like craters were observed. In addition, the lips and ridges formed during cutting or plastic deformation provided the important sources for platelet formation.

\subsubsection{Subsurface appearance}

The SEM micrographs of the subsurface of the two specimens (68\% and 3\% carbide content), that were erosion tested using $8000 \mathrm{~g}$ of particles at different incident angles of $\pi / 18, \pi / 4.5$, and $\pi / 2$, are respectively shown in Figs. 10(a) to $\sim(f)$. For low angle erosion, the eroded subsurface of $3 \%$ carbide specimen had plastic deformation with lip shape tearing along the erosive direction; the eroded subsurface of $68 \%$ carbide specimen, owing to the highest hardness and the lowest impact toughness, only had a few lips shape and minor cracking. For medium angle erosion, the eroded subsurface of $3 \%$ carbide specimen showed the appearance of deeper smear crater and plastic deformation on the "bull's eye" structure. Under high angle erosion, the subsurfaces of all specimens showed signs of crevice erosion. Some of the
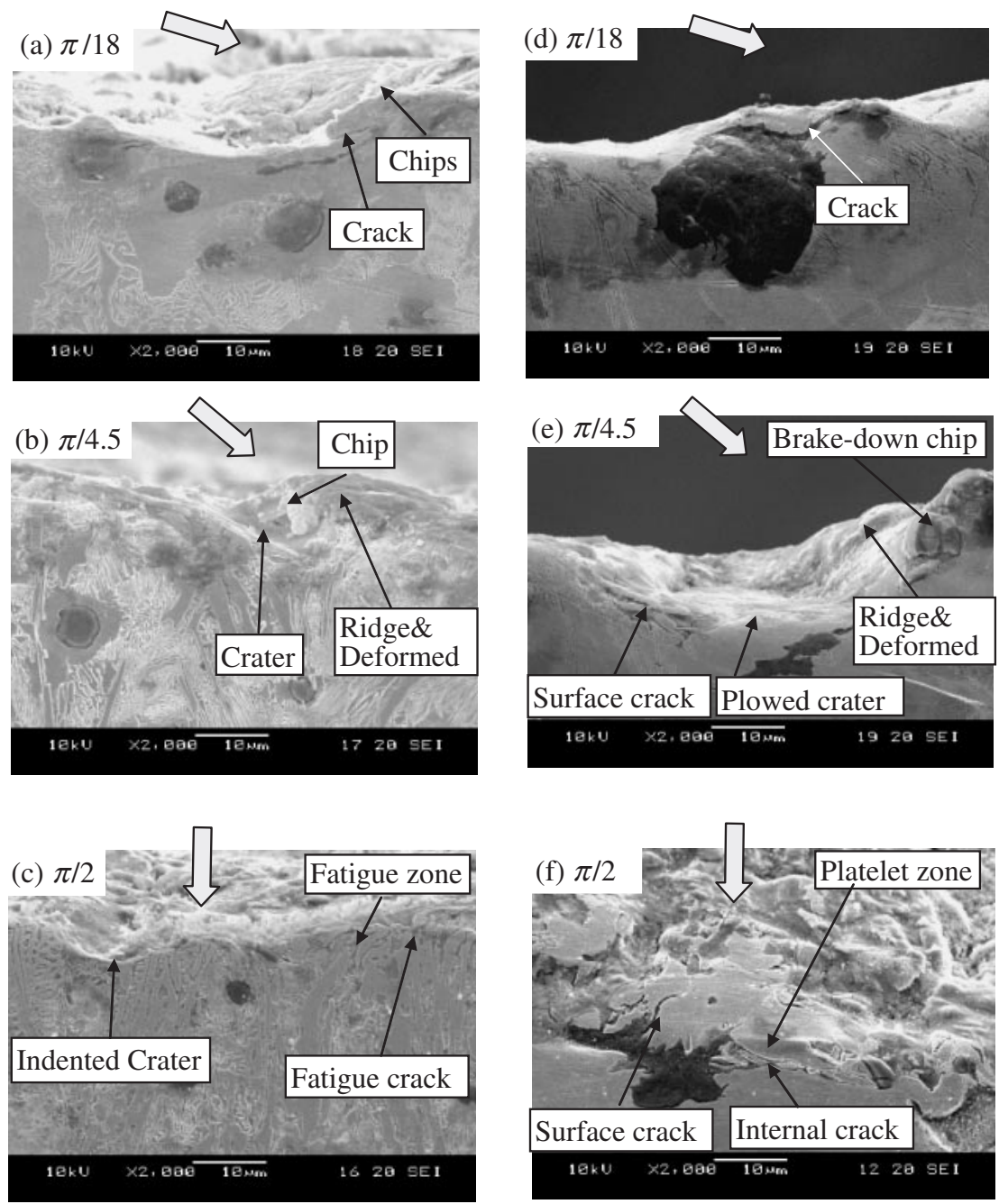

Fig. 10 SEM observation of subsurface after $8000 \mathrm{~g}$ repetitious erosion; (a) $68 \%-\pi / 18$, (b) $68 \%-\pi / 4.5$, (c) $68 \%-\pi / 2$, (d) $3 \%-\pi / 18$,

(e) $3 \%-\pi / 4.5$, (f) $3 \%-\pi / 2$, where the arrows indicate incident direction projected on surface. 
cracks extended from the surface to the subsurface and caused the material to break down by normal force. This mechanism created fatigue cracks and internal cracks of four specimens at $\pi / 2$ impinged angle where the highest normal force exists, The highest normal force explained why impinged at $\pi / 2$ created bigger damage than at $\pi / 2.25$. It revealed the erosion morphology to be a ductile-brittle fracture.

\section{Conclusions}

The effects of eutectic carbides (cementite) on erosion behavior of ductile cast iron were studied. The following conclusions were made:

(1) The amount of the eutectic carbides increase with increasing cooling rate and low silicon content, while the ferrite content decrease accordingly. However, pearlite did not seem to play the role of balancing ferrite content in the matrix of ductile iron. Instead, eutectic carbide did. Thus, carbide (or alternatively ferrite) was the controlling factor of the properties obtained in this investigation.

(2) The erosion rate of all the ductile iron in this experiment seems to be increasing from a impinged angle of $\pi / 18$ to $\pi / 4.5$ and then started to decline from an angle of $\pi / 4.5$ to $\pi / 2.25$ before it picked up again from $\pi / 2.25$ to $\pi / 2$. Erosion rate also was found in the same trend that higher carbide content yielded better erosion resistance and lower rates.

(3) The maximum erosion rate occurred at an erosion incident angle of $\pi / 4.5$, and the deepest erosion penetration occurred at an erosion incident angle of $\pi / 3.6$. This mechanism created fatigue cracks and internal cracks of four specimens at $\pi / 2$ impinged angle where the highest normal force exists. The highest normal force explained why impinged at $\pi / 2$ created bigger damage than at $\pi / 2.25$. It revealed the erosion morphology to be a ductile-brittle fracture.

(4) The morphology of the eroded surface at high eutectic carbide content specimen was found to include: short and shallow cutting at low incident angle erosion; cutting and smear craters at medium incident angle erosion; and indented craters and surface cracking at high incident angle erosion. For low eutectic carbide content specimen the following was found: deep and wide plowing at low incident angle erosion; deformation and smear craters at medium incident angle erosion; and indented craters at high incident angle erosion.

\section{Acknowledgements}

The authors wish to thank The National Science Council (Taiwan, Republic of China) for the financial support granted under Contract No. NSC89-2216-E-036-032.

\section{REFERENCES}

1) W. J. Evans, S. F. Carter, Jr. and J. Wallace: AFS Trans. 89 (1981) $293-$ 321.

2) K. Shimizu, T. Noguchi, T. Yamaguchi and T. Kamada: AFS Trans. 102 (1994) 285-289.

3) ASM metal handbook: Friction, lubrication, and wear technology, 18 (1992) pp. 199-213.

4) A. Magnee: Wear 181-183 (1995) 500-510.

5) Y. I. Oka, H. Ohnogi, T. Hosokawa and M. Mattsumura: Wear 203-204 (1997) 573-579.

6) I. Finnie, A. Levy and D. H. McFadden: ASTM STP 66436 (1979).

7) J. G. A. Bitter: Wear 6 (1963) 5-21.

8) J. G. A. Bitter: Wear 6 (1963) 169-190.

9) K. H. Zum Gahr: tribology, series No 10, Amsterdam (1987) pp. 531553.

10) F. Y. Hung, L. H. Chen and T. S. Lui: Mater. Trans. 42 (2001) 26132621.

11) F. Y. Hung, L. H. Chen and T. S. Lui: Mater. Trans. 43 (2002) 42-48.

12) J. J. Kim and S. K. Park: J. Mater. Sci. Lett. 17 (1998) 1503-1506.

13) P. A. Engel: Impact Wear of Materials, (Elsevier, 1976).

14) A. W. Ruff and S. M. Wiederhorn: Treatise on Mat. Sci. and Tech., in: C.M. Preece (Ed.), Vol. 3, 16, (1979) p. 69.

15) ASTM A327-91: Standard Test Methods for Impact Testing of Cast Irons $^{l}$, (1996) pp. 148-153.

16) H. Y. Teng, C. H. Hsu, S. C Chiu and D-C Wen: Mater. Trans. 44 (2003) 1480-1487.

17) ASTM G76-95: Standard Test Method for Conducting Erosion Tests by Solid Particle Impingement Using Gas Jets' (1997) pp. 305-309.

18) K. V. Pool, C. K. H. Dharan and I. Finne: Wear 107 (1986) 1-12.

19) F. Y. Hung, L. H. Chen and T. S. Lui: Wear 252 (2002) 985-991.

20) G. P. Tilly and W. Sage: Wear 16 (1970) 447-465.

21) J. W. Edington and I. G. Wright: Wear 48 (1978) 131-144.

22) C. M. Preece and N. H. Macmillan: Annu. Rev. Mater. Sci. 95 (1977) 12.

23) S. I. Karsay: Ductile Iron Production Practices, (Des Plaines, IL: American Foundrymen's Society, 1979) pp. 54-64.

24) S. I. Karsay: Modern Casting, (1975) 204-213.

25) J. S. Hansen: Relative Erosion Resistance of Several Metals, Erosion: Prevention of Useful Applications, STP 664, AST 1979, pp. 148-162.

26) G. A. Sargent, M. K. Keshavan, P. K. Mehrotra and H. Conrad: The Erosion of Plain Carbon Steels by Ash Particles From a Coal Gasifier, Proceeding of Internationational Conference on Wear of Materials, (American Society of Mechanical Engineers, 1981), pp. 613-618.

27) L. G. Peterson, G. A. Sargent and H. Conrad: Effect of Microstructure on the Erosion of Steel by Solid Particles, Proceedings of International Conference on Wear of Materials, (American Society of Mechanical Engineers, 1985), pp. 661-671. 